\title{
Model of Sport Progress Based on Strategic Performance Test: Moderating Role of Self-Compassion
}

\begin{abstract}
Introduction: Self-Compassion as a powerful source of regulating and controlling of athlete's emotions behavior has wide application. Therefore, the purpose of this research was examining the model of sport progress based on strategic performance test with moderating role of self-compassion.

Methods: The present study is of the correlation type. The statistical population included all professional athletes in Golestan province, which 301 athlete were selected as a statistical sample by simple random sampling method. The research instruments included Thomas Strategic Performance Test Questionnaire (TOPS) and a modified version of the Self-Compassion Scale (SCC). SPSS software and AMOS were used to analyze the data.

Results: The results showed that sports performance had a significant effect on self-compassion $(\beta=0.33, \mathrm{P} \leq 0.01)$ and selfcompassion on sports success $(\beta=0.54, \mathrm{P} \leq 0.01)$.

Conclusion: Therefore, considering that self-compassion had a significant effect on sports performance, so it is suggested that the use of compassion to deal with the consequences of negative thoughts and motivate athletes to increase the range of championship sports.
\end{abstract}

Keywords: Test of Performance Strategic, Self-Compassion, Sports Progress

\section{Article Info}

Authors:

Ghezelsoflou HR ${ }^{1 \bullet}$

Alavi $\mathrm{SH}^{2 *} \odot$

Received: 2020.10.31

Accepted: 2020.11.20

E-Published: 2020.12.05

\footnotetext{
Affiliations

1- Assistant Professor, Department of sport management, Gonbad Kavous University, Gonbad Kavous, Iran.

2- Assistant Professor, Physical Education and Sport Sciences, Department of Technical and Vocational of Mahmoud Abad Branch of Technical and Vocational University (TVU), Mazandaran, Iran.

Corresponding Author: shalavi@,tvu.ac.ir
}

\section{Citation Subjects:}

Ego, Personality, Caring, Exercise, Athletics, Exercise Movement Techniques, Exercise Therapy, Feelings, Regret, Acceptance Process 
فصلنامه يֶرستار و يزشك در رزم / پاييز 99 / سال هشتم

الكَى پيشرفت ورزشى برمبناى آزمون عملكرد راهبردى: نقش ميانجى شفقت با خود

\begin{tabular}{|c|c|}
\hline اطلاعات مقاله & جحكيده \\
\hline سميدرضا قزلسندان: & 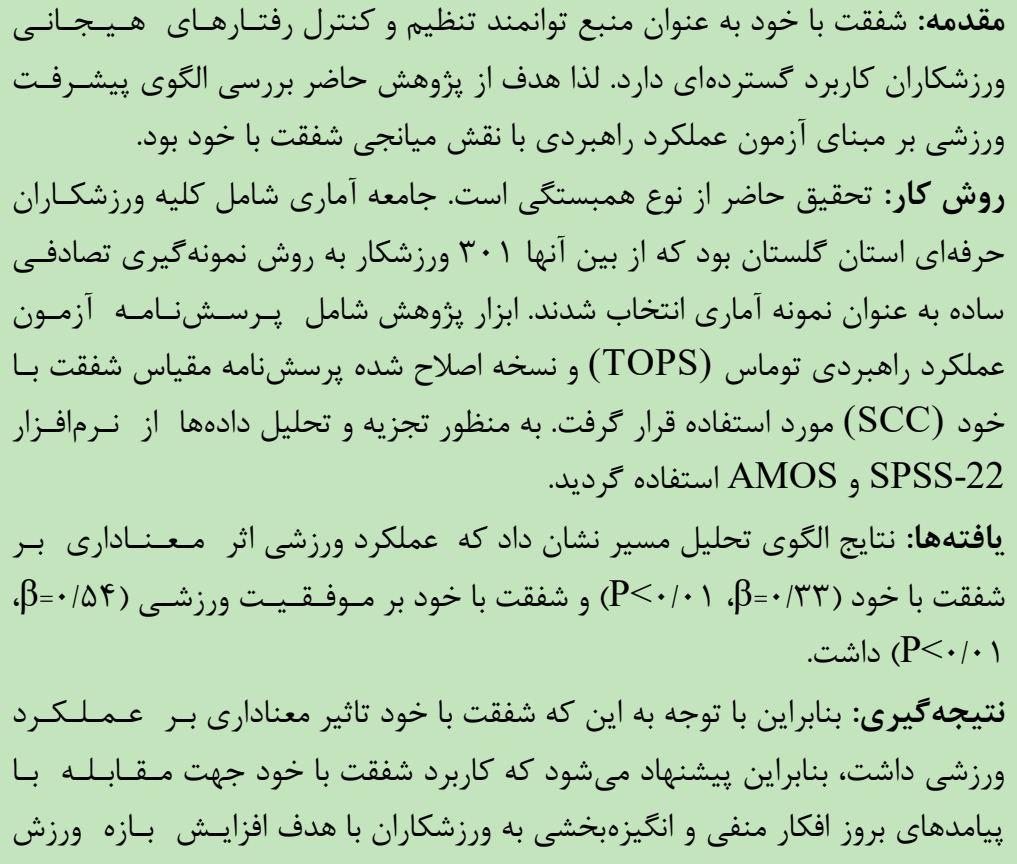 \\
\hline 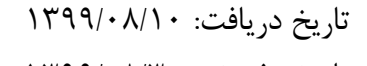 & قهرمانى مد نظر قرار كيرد. \\
\hline 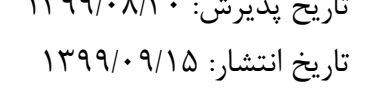 & وازگان كليدى: آزمون عملكرد راهبردى، يِشرفت ورزشى، شفقت با خود \\
\hline
\end{tabular}

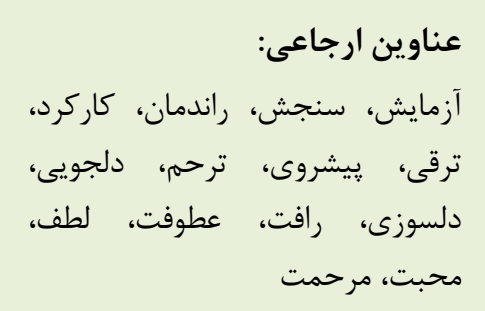
وابستخى سازمانى نويسندكان
1- استاديار، مديريت ورزشى دانشخاه كنبد كاووس، كنبد كاووس، ايران.

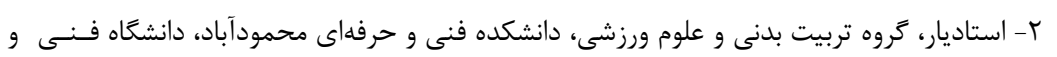

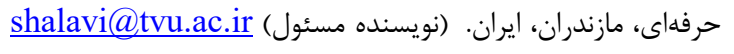




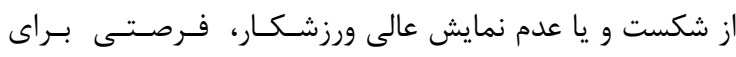

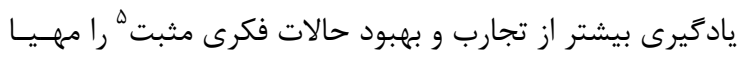

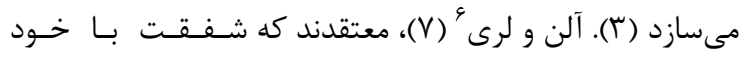

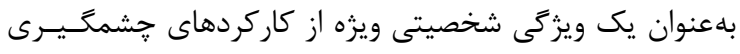

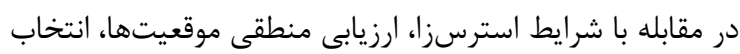

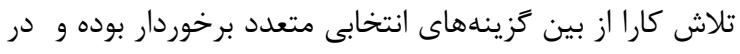

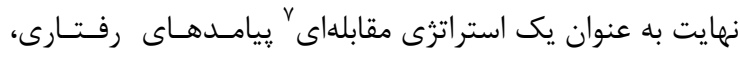
عاطفى و ادراكى موثرى را ايجاد مى نمايد.

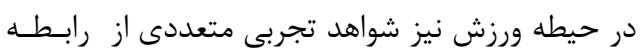

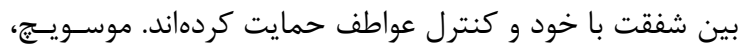

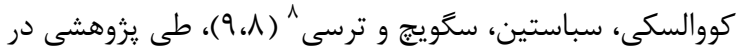

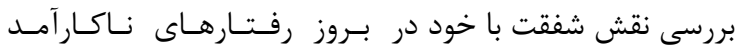

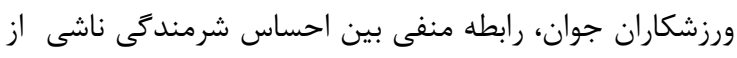

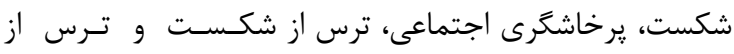

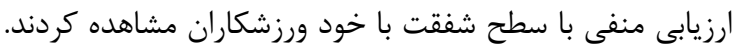

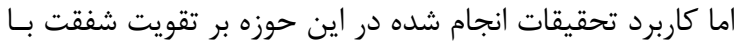

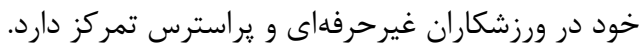

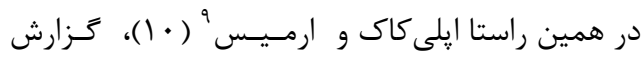

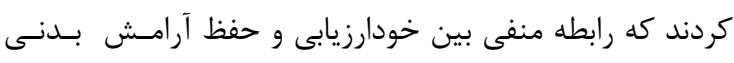

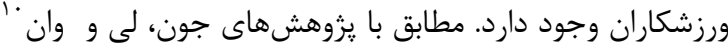

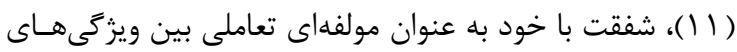

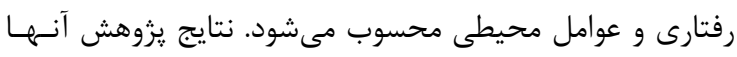

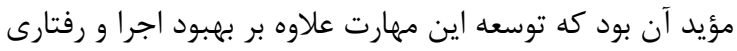

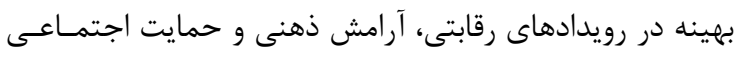

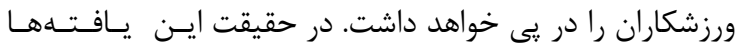

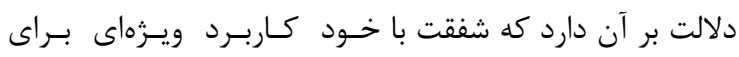

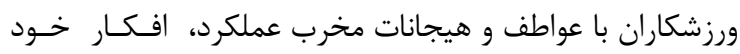

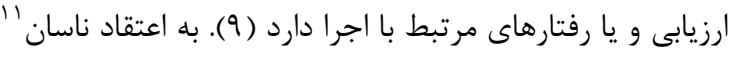

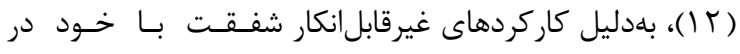

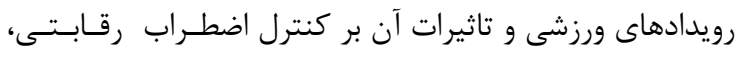
تنظيم احساسات، خودكارآمدى و ترس از شكست ورزشــــــاران،

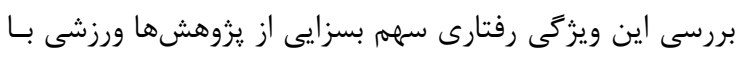

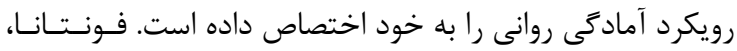

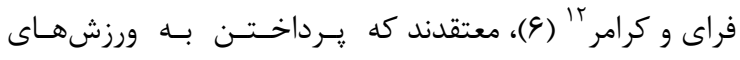

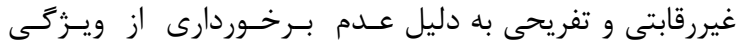

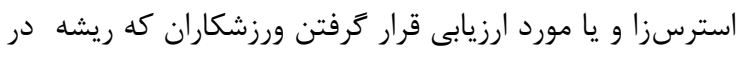

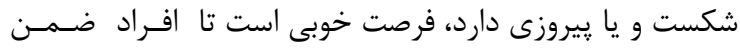

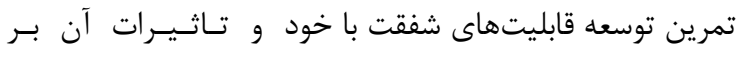

5- Positive State of Mind

6- Allen and Leary

7- Coping Resource Strategy

8- Mosewich, Kowalski, Sabiston, Sedgwick \& Tracy

9- Epli Koç \& Ermiş

10- Jeon, Lee \& Kwon

11- Nathan

12- Fontana, Fry \& Cramer
مقدمه

موفقيت ورزشى به بررسى جنـد بعدى و تعاملى همهجــانـبــهـ از

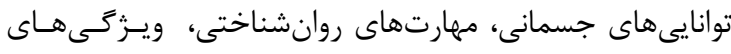

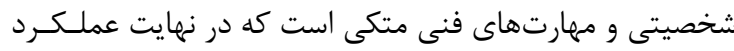

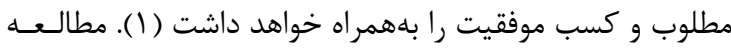

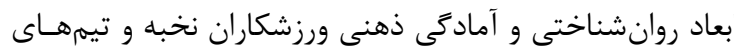

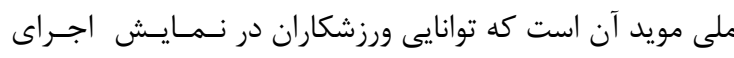

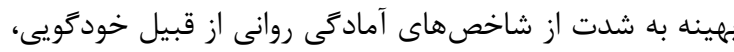

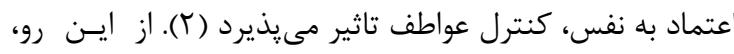

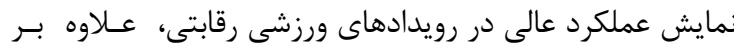

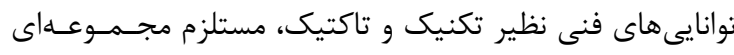

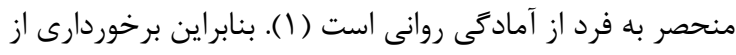

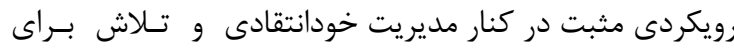

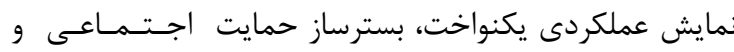

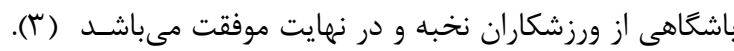

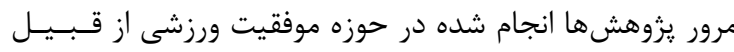

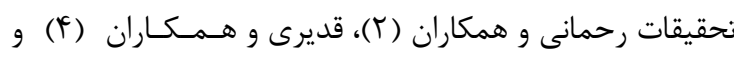

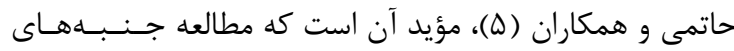

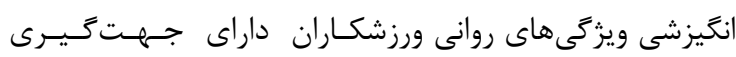

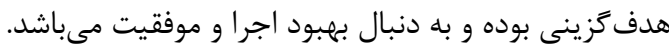

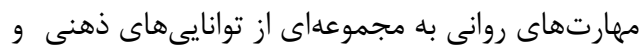

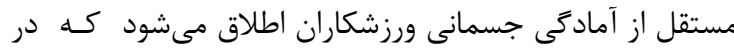

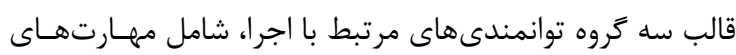

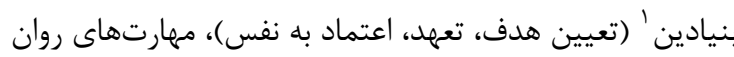

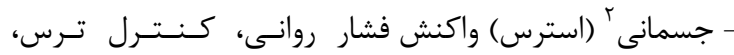

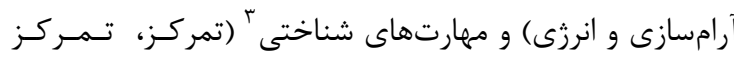

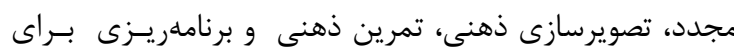

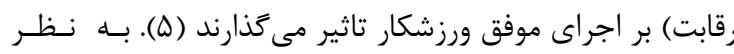

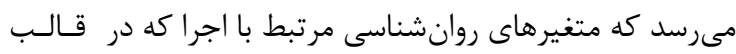

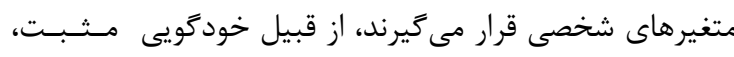

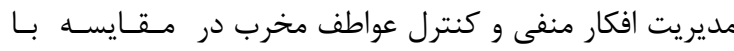

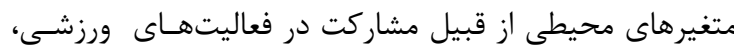

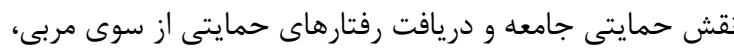

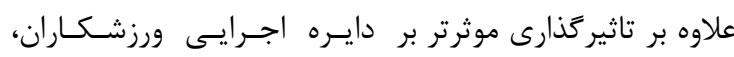

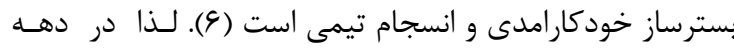

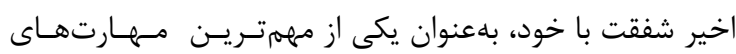

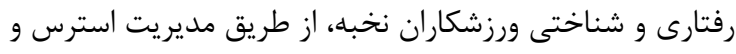

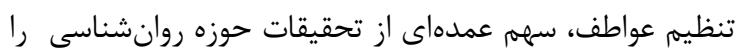

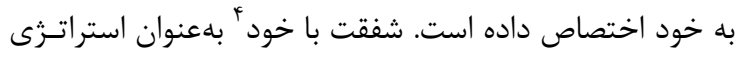
كارايى است كه علاوه بر تعديل رويكردهاى ذهنى منفى ناشسى

1- Foundation Skills

2- Psychosomatic Skills

3- Cognitive Skills

4- Self-Compassion 
هدف

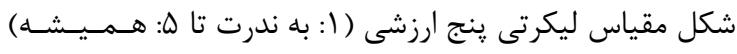

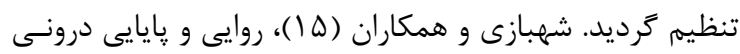

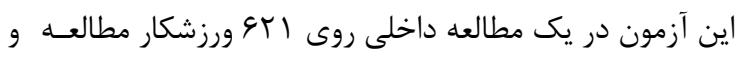

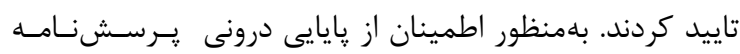

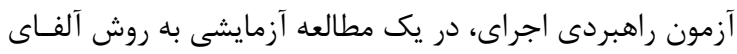

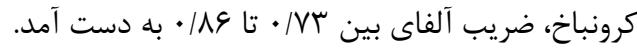

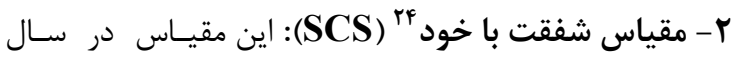

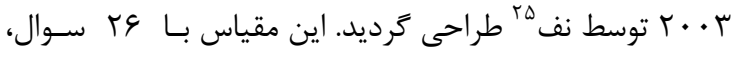

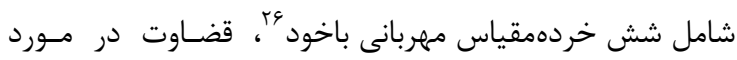

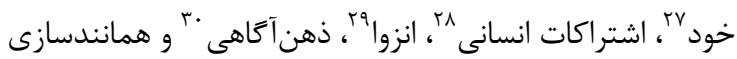

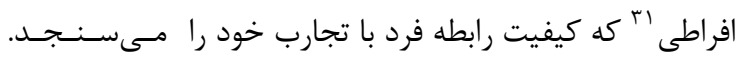

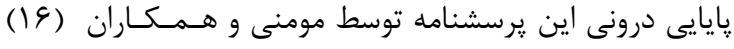

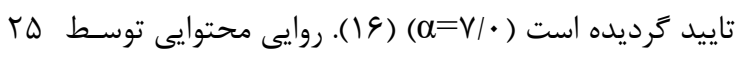

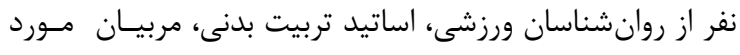

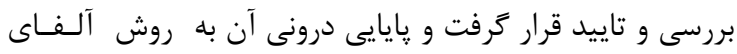

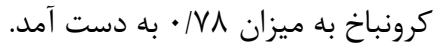

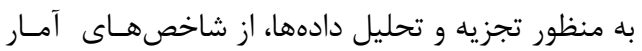

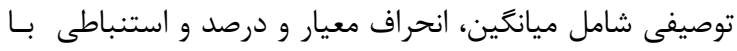

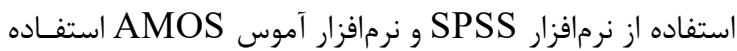

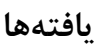

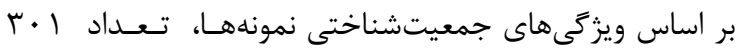
ورزشكار با ميانكين سنى (

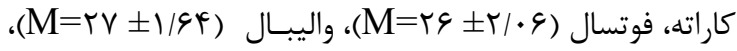
بسكتبال (M (M=

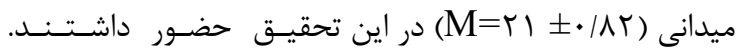

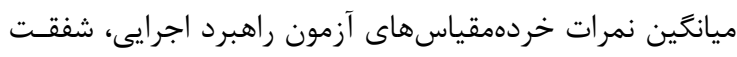

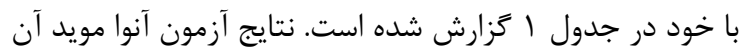

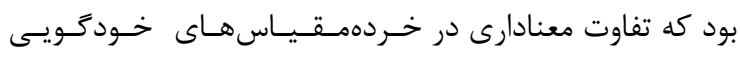

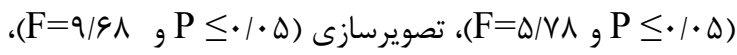
آرامسازى (م) (P

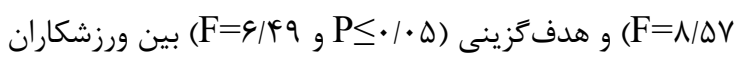

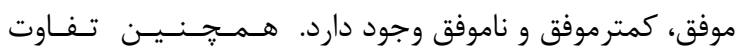

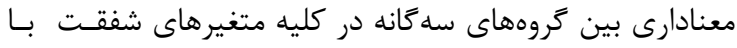
خود مشاهده گرديد (ه • (P

21- Minnesota Living With Heart Failure Questionnaire 22- Green, Porter, Bresnahan \& Spertus

23- Concentration

24- Self-Compassion Scale - SCS

25- Neff

26- Self-Kindness

27- Self-Judgment

28- Common Humanity

29- Isolation

30- Mindfulness

31- Over-Identification
احساس شرمندگى و غرور، فضاى انخَيزشى ايجاد شده از سـوى

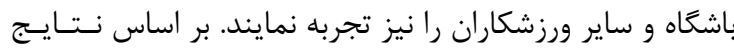

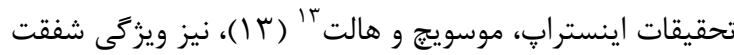
با خود از قابليت توسعه و آموزش برخورابن موردار است. بنابراين لازمه

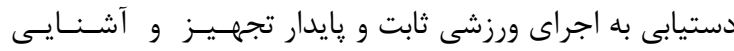

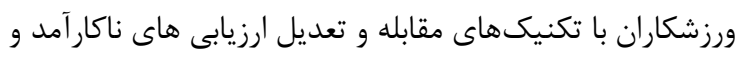

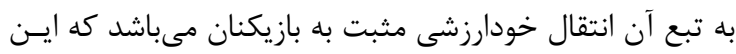

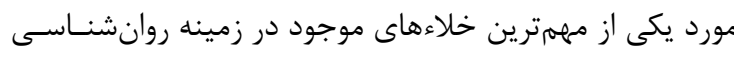

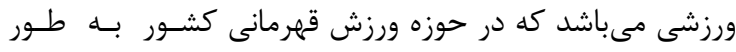

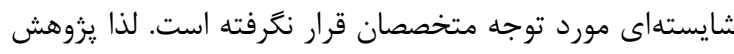

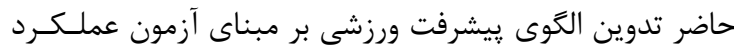
راهبردى با ميانجى گرى شفقت با خود مى بـاشد.

روش كار

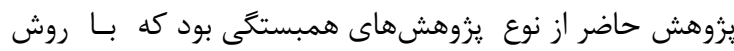

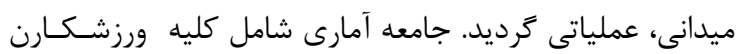

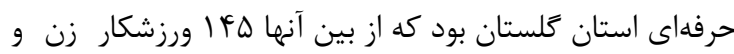

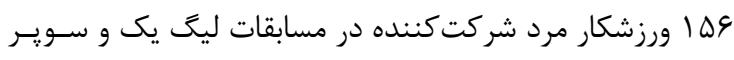

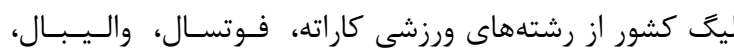

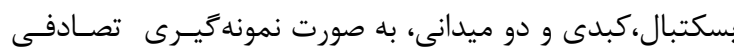

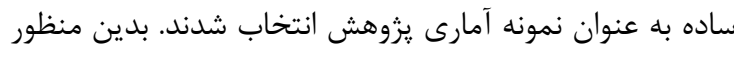

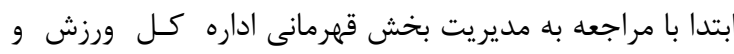

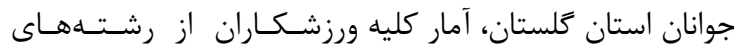

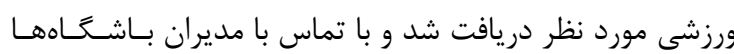

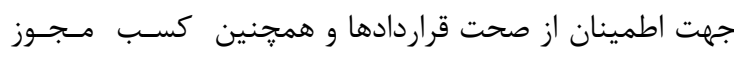

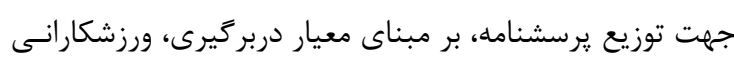

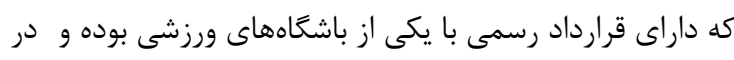

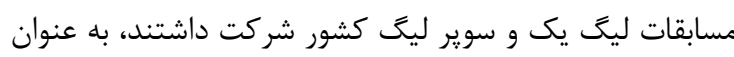

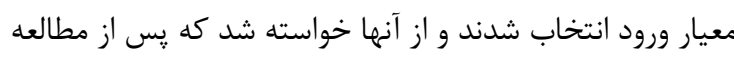

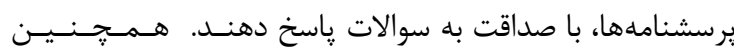

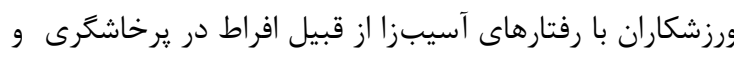

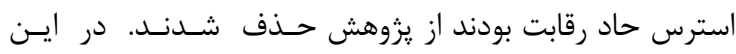

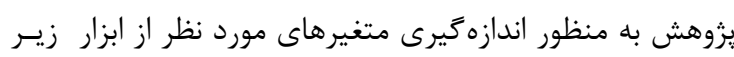

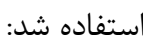

1- آزمون عملكرد راهبردى "

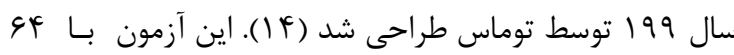

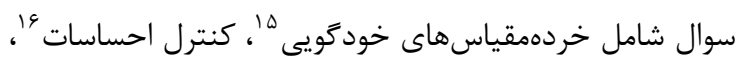

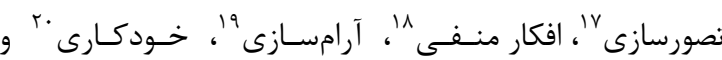

13- Ingstrup, Mosewich, \& Holt

14- Test of Performance Strategic - TOPS

15- Self-Talk

16- Emotional Control

17- Imagery

18- Negative Thinks

19- Relaxation

20- Automaticity 


\section{جدول ا: نتايج ميانكَين نمرات عملكرد راهبردى و شفقت با خود ورزشكاران و آزمون آنوا}

\begin{tabular}{|c|c|c|c|c|}
\hline & موفق & كمتر موفق & ن ناموفق & \\
\hline خردهمقياسها & $\mathrm{M} \pm \mathrm{sd}$ & $\mathrm{M} \pm \mathrm{sd}$ & $\mathrm{M} \pm \mathrm{sd}$ & $\mathrm{F}$ \\
\hline خودكويى & $\Lambda / I \pm V I / r \mu$ & $f / l \pm V / / . q$ & $F / r \pm 11 / 11$ & $\Delta / V \Lambda^{*}$ \\
\hline كنترل عواطف & $V / \cdot \pm \Delta q / \wedge r$ & $\Delta / 1 \pm \wedge \varepsilon / F q$ & $\Delta / 1 \pm \cdot F / \Lambda \varphi$ & $V / r q$ \\
\hline تصويرسازى & $|r /| \pm \Delta \mid / \Delta r$ & $\wedge / \cdot \pm 91 / \wedge 9$ & $q / r \pm \cdot r / \backslash \Lambda$ & $9 / 9 \Lambda^{*}$ \\
\hline افكار منفى & $\Delta / \cdot \pm r \mid / V \Delta$ & $9 / 1 \pm \Lambda 1 / \Delta \Delta$ & $9 / 1 \pm \lambda r / r r$ & F/r \\
\hline آرامش & $|r /| \pm F N|G|$ & $\| / / \pm \Lambda N / \varepsilon V$ & $|1 / 1 \pm r \varepsilon| \wedge \mid$ & $\Lambda / Y^{*}$ \\
\hline خودكارى & $|r /| \pm q r / r q$ & $\Lambda \cdot \pm \cdot r / \Lambda r$ & $V / I \pm r \Lambda / G Y$ & $\Lambda / \Delta V^{*}$ \\
\hline هدف گزينى & $\| / 1 \pm Y \Lambda / F V$ & $11 / \cdot \pm r r / 9 r$ & $1 \cdot / 1 \pm \Phi \mu / \& V$ & $9 / 4 q^{*}$ \\
\hline مهربانى با خود & 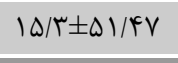 & $\mid T / I \pm Y N / F T$ & $1 \cdot / r \pm r \Lambda / F V$ & $11 / 4 \Lambda^{*}$ \\
\hline قضاوت خود & $q / 1 \pm r \Lambda / F V$ & $\| / r \pm r \Lambda / \mathcal{E V}$ & $|r / T \pm| N / \mid V$ & $\mid r / \cdot V^{*}$ \\
\hline اشتراكات انسانى & $\mid Q / r \pm \cdot / \cdot V$ & $\mid r / r \pm r \Lambda / F V$ & $1 \cdot / r \pm r N / I r$ & N/צ"* \\
\hline انزوا & $N / r \pm r \mid / r V$ & $1 \cdot / r \pm \varepsilon N / \cdot r$ & $|r / T \pm| N \mid \cdot V$ & $\mid 9 / \Lambda e^{*}$ \\
\hline ذهن آكاهى & $\mid F / T \pm Y N / F Y$ & $|r / r \pm 9 r / 1|$ & $\| / r \pm \cdot N / V V$ & $11 / \pi r^{*}$ \\
\hline فزون همانندسازى & $V / I \pm \cdot N / F V$ & $9 / r \pm r \Lambda / / \Lambda$ & $11 / r \pm \wedge \mathrm{N} / 11$ & $9 / V \varphi^{*}$ \\
\hline $\mathrm{p} \leq \cdot / \cdot \Delta^{*}$ & & & \multicolumn{2}{|c|}{ سطح معنادارى } \\
\hline
\end{tabular}

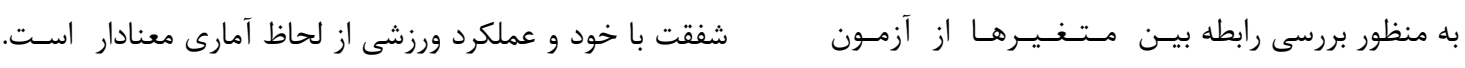

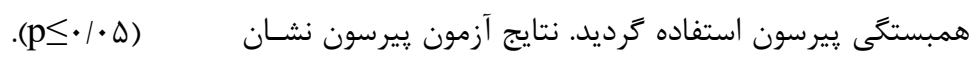

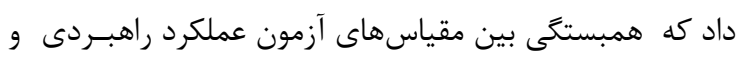

جدول r: ماتريس همبستكى متغيرهاى يزوهش

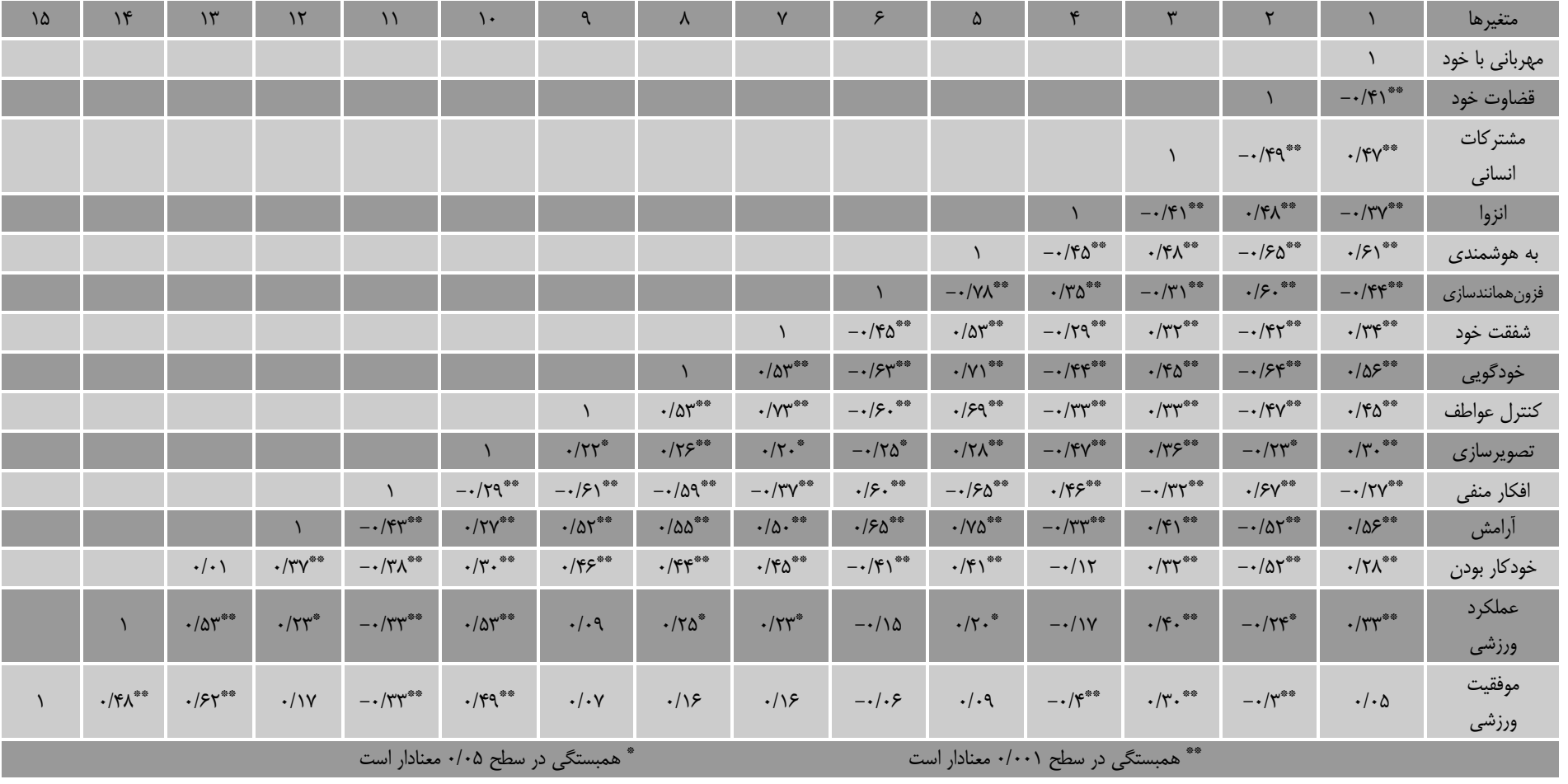

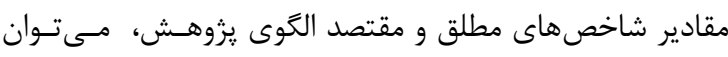
اظهار داشت كه الكوى يزوهش از برازش بسيار مطلوب برخوردار بردار

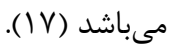

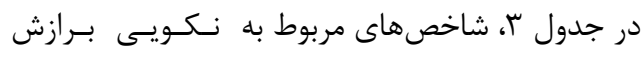

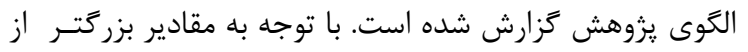

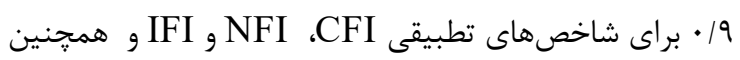


جدول rا: شاخص هاى مربوط به نكويى برازش الكوى يزوهش

\begin{tabular}{|c|c|c|c|c|c|c|}
\hline شاخصهاى مقتصد & \multicolumn{3}{|c|}{ شاخصهاى تطبيقى } & \multicolumn{2}{|c|}{ شاخصهاى مطلق } & شاخصها/مدل ها \\
\hline RMSEA & IFI & CFI & NFI & df $/ \chi^{r}$ & GFI & \multirow{2}{*}{ برآورد } \\
\hline$\cdot / \cdot r q$ & .194 & $\cdot / 99$ & .194 & $1 / 9 \mathrm{~V}$ & $\cdot / 9 V$ & \\
\hline$<\cdot 11$ & $>\cdot 19$ & $>\cdot 11$ & $>\cdot 19$ & $\leq r$ & $>\cdot 19$ & دامنه مورد قبول \\
\hline تاييد & تاييد & تاييد & تاييد & تاييد & تاييد & نتيجه برازش مدل \\
\hline
\end{tabular}

نتايج تحليل مسير الكوى يزووهش در شكل شـــاره

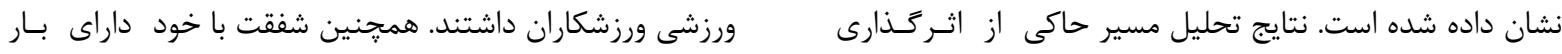

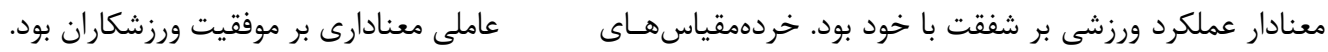

شكل ا: تحليل مسير الكوى تفهيمى يزوهش براى بررسى ارتباط بين عملكرد ورزشى، شفقت خود و موفقيت ورزشى

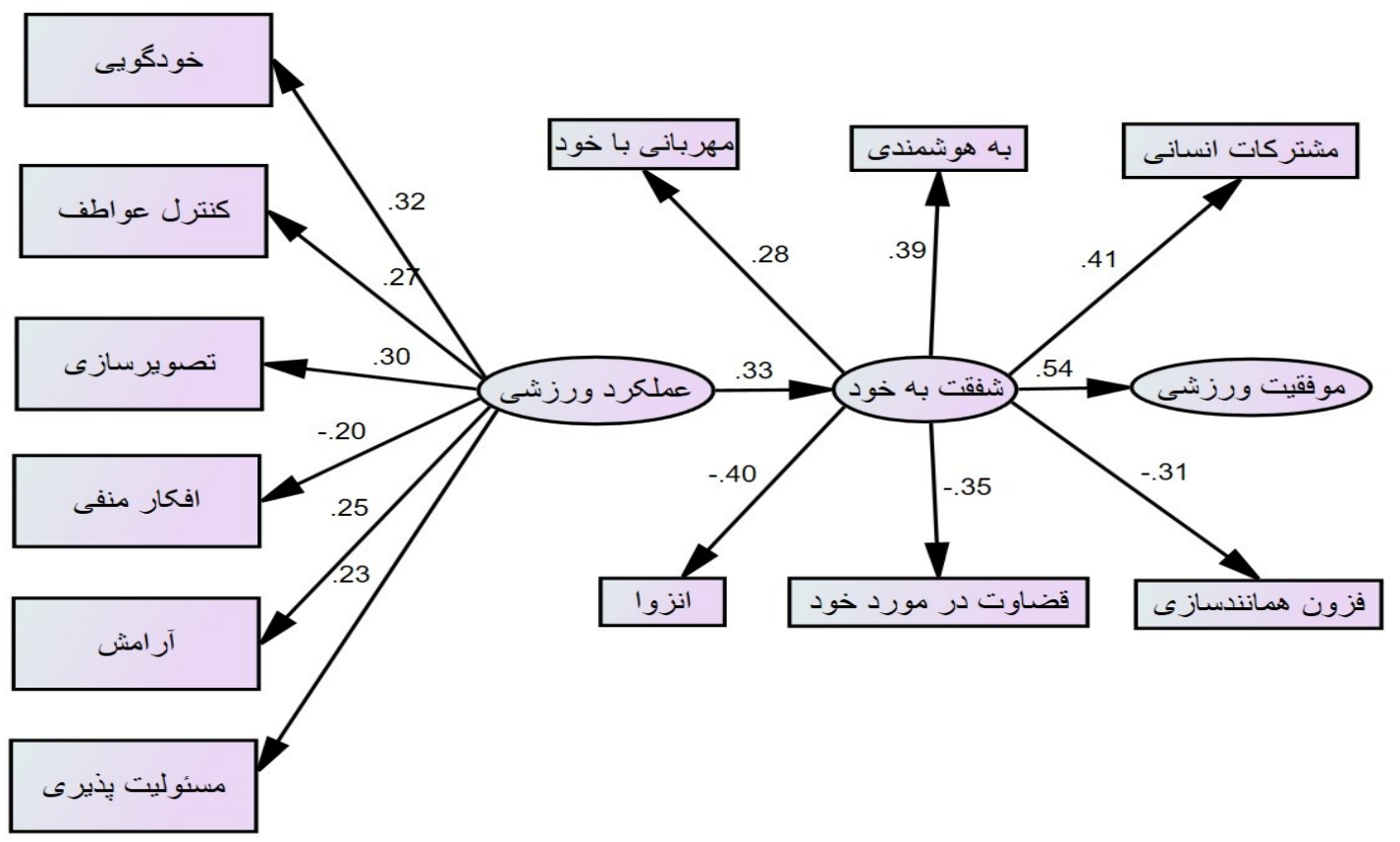

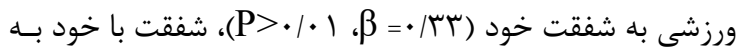

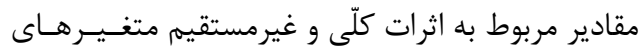

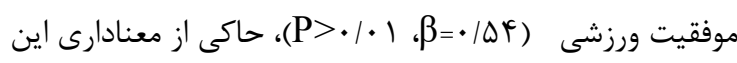

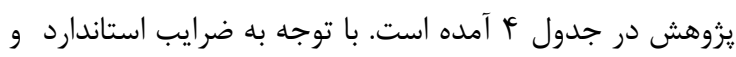
ضرايب مىباشد.

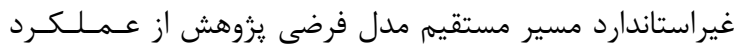

جدول f: ضرايب استاندارد و غيراستاندارد متغيرها بر مبناى ميانجى

\begin{tabular}{|c|c|c|c|c|c|}
\hline P-value & C.R & S.E & $\beta$ & B & مسيرها ـــ \\
\hline$<\cdot / \cdot \cdot 1$ & $F / \Delta \Delta$ &.$/ \cdot r$ & זr/. & $\cdot 110$ & عملكرد ورزشى به شفقت خود \\
\hline$<\cdot / \cdot \cdot 1$ & $1 \cdot 19$. & $\cdot / \cdot 4$ & $\cdot \mid \Delta F$ & $\cdot 149$ & شفقت خود به موفقيت ورزشى \\
\hline
\end{tabular}

موفقيت ورزشى ورزشكاران تاثير گذار بودند. ايسن نـتـايسج در

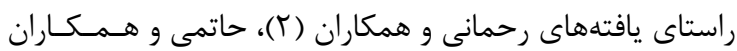

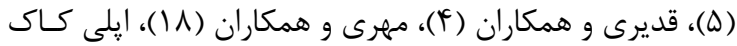

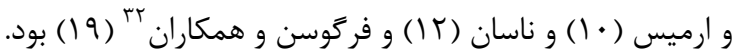

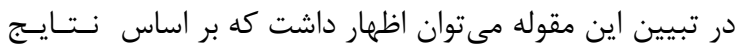

\section{بحث و نتيجه} هدف از يزوهش حاضر بررسى الكوى يِيشرفت ورزشى برى بر مبناى

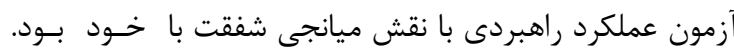
يافتههاى يزوهش نشان داد كه خردهمقياسهاى آزمون عملكرد

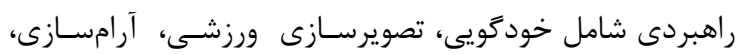

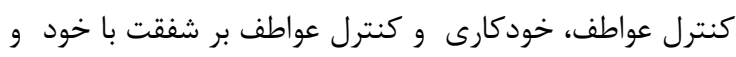


به آموزش، يادكيرى و انتقال آن ايجاد نمايند. لذا مسىتسوان از

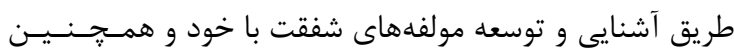

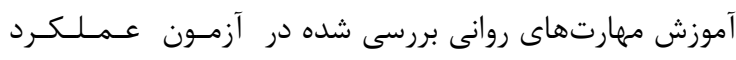

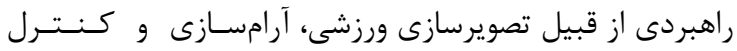

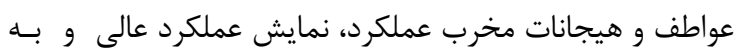

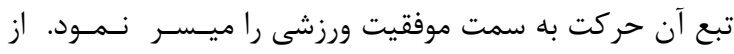

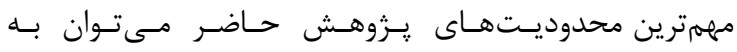

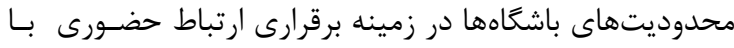

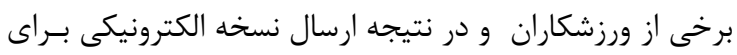

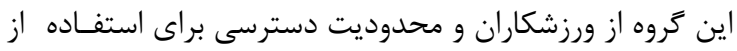

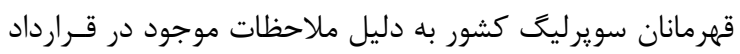

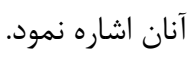

\section{تشكر و قدردانى}

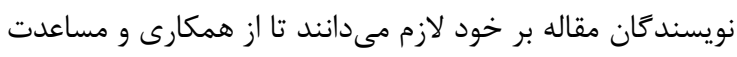

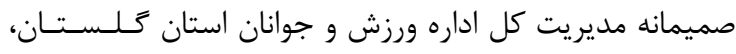

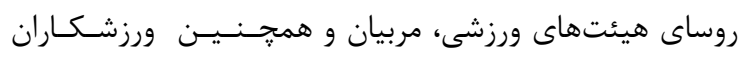
شركت كننده در اين يزوهش كمال تشكر را داشته باشند.

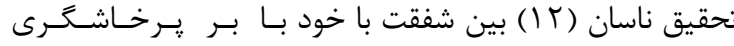

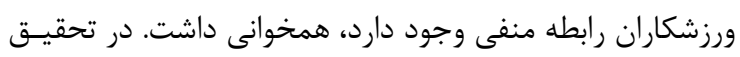

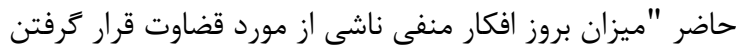

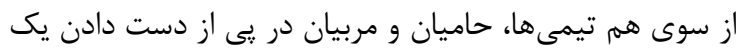

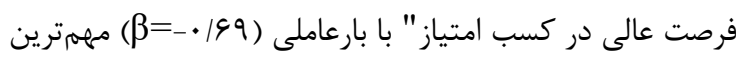

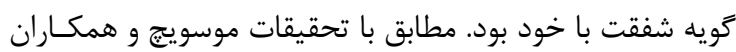

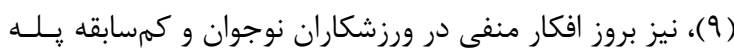

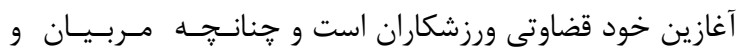

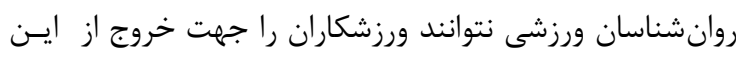

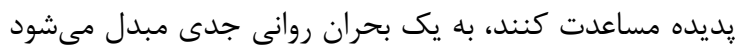

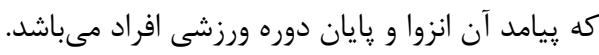

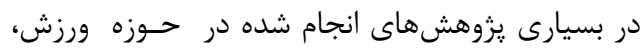

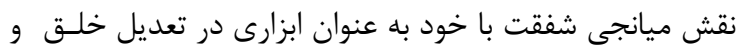

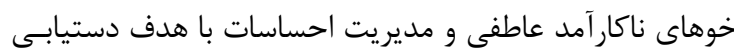

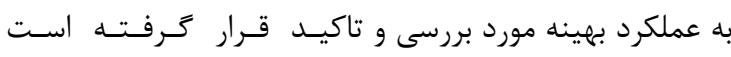

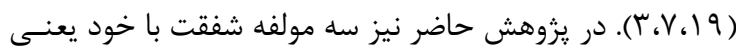

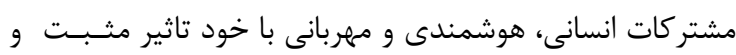

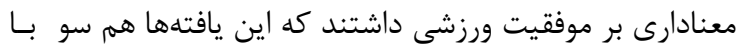

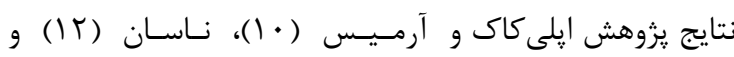

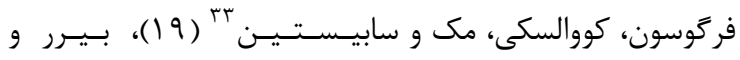

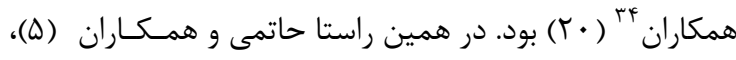

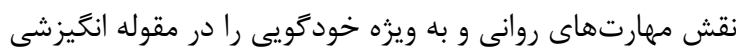

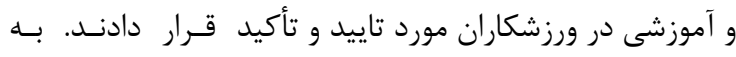

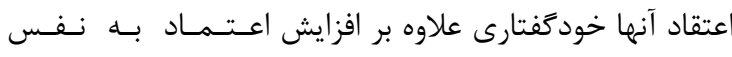

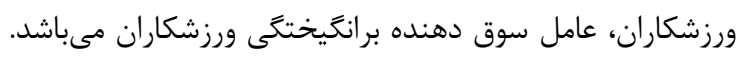

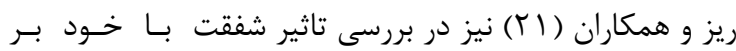

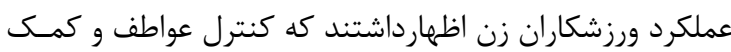

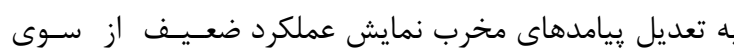

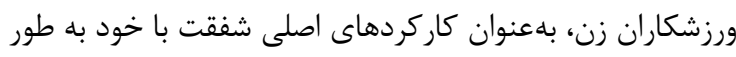

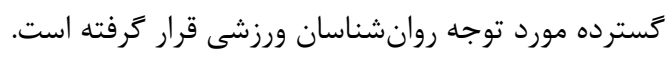

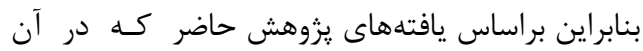

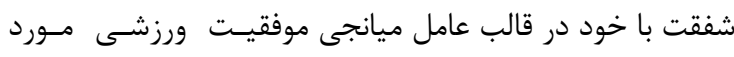

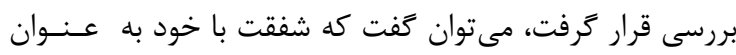

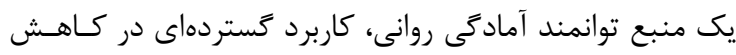

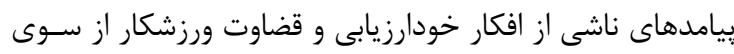

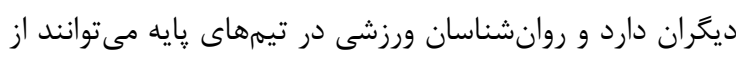

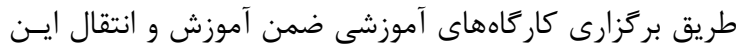

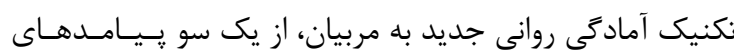

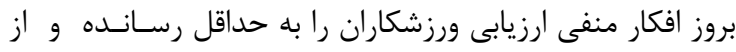

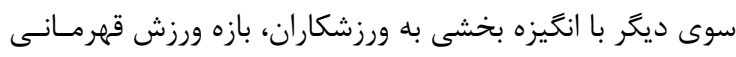

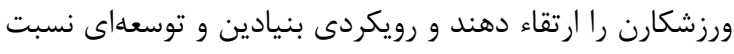

33- Kowalski, Mack \& Sabiston 34- Birrer 


\section{References}

1- VaezMousavi, M., Mousavi, A. Effective factors in sport achievement based on related theories and models. Journal of Sport Psychology Studies, 2015, 10: 25-50.[Persian]

2- Rahmani, M., Bahari, M., Ahmadi, P. Comparison of Mental Skills of Athletes of the Iranian National Team in Asian Games in 2006 and 2018. Sport Psychology Studies, 2020; 9(33): 1-18. doi: 10.22089/spsyj.2020.7027.1752.

3- Amber D. Mosewich., Peter R.E. Crocker, Kent C. Kowalski, \& Anita DeLongis. Applying selfcompassion in sport: An intervention with women athletes. Journal of Sport \& Exercise Psychology, 2013, N 35: 514-524.

4- Ghadiri, F., Nemati, M., Frouzandeh Hafshejani, Z. The Comparision of Mental Skills of elite male Wushu and Kickbox Players. Sport Psychology Studies, 2018; 7(23): 17-34. doi: 10.22089/ spsyj.2018.1206.[Persian]

5- Hatami, F., Tahmasbi, F., Shayan, S. The Effects of Instructional and Motivational Self-Talk on Anxiety, Self-Confidence and Performance of Badminton Long Serve. Sport Psychology Studies, 2018; 7(24): 17-34. doi: 10.22089/ spsyj.2018.4709.1503.[Persian]

6- Fontana Mario. S., Fry, M. D., \& Cramer, E. Exploring the Relationship between Athletes' Perceptions of the Motivational Climate to Their Compassion, Self-Compassion, Shame, and Pride in Adult Recreational Sport. Measurement in Physical Education and Exercise Science, 2017; 21(2): 101111.

7- Allen, A.B., Leary, M.R. Self-compassion, stress, and coping. Social and Personality Psychology Compass, 2010; 4:107-118. PubMed doi:10.1111/j.1751-9004.2009.00246.

8- Mosewich, A.D., Kowalski, K.C., Sabiston, C.M., Sedgwick, W.A., \& Tracy, J.L. Selfcompassion: A potential resource for young women athletes. Journal of Sport \& Exercise Psychology, 2011, 33: 103-123.

9- Mosewich, A.D., Crocker, P.R.E., \& Kowalski, K.C. Managing injury and other setbacks in sport: Experiences of (and resources for) high performance women athletes. Qualitative Research in Sport, Exercise, and Health. 2013, 4 (11): 11-29. doi:10.1080/2159676X.2013.766810.

10- Epli Koç, H., \& Ermiş, E. Self-compassion as a predictor of social physique anxiety in athletes. Journal of Human Sciences, 2016; 13(3): 5214-22. doi:10.14687/jhs.v13i3.4120.

11- Jeon Hyenso., Lee, K., \& Kwon, S. Investigation of the structural relationships between social support, self-compassion, and subjective wellbeing in Korean elite student athletes. Psychological reports, 2016; 119 (1): 39-54.

12- Nathan P. Lee. The relationship between mindful self-compassion pre-competition, facilitative state-anxiety of NCAA women's equestrian team athletes. A thesis master of arts in kinesiology in the college of Health and Human Services California state university, Fresno. 2015; 11-13.
13- Ingstrup Megan. S., Mosewich, A. D., \& Holt, N. L. The development of self-compassion among women varsity athletes. The Sport Psychologist, 2017; 31(4): 317-331.

14- Thomas, P .R, Murphy, S.M.Test of performance strategies: development and preliminary validation of a comprehensive of athlete's psychological skills. Journal of sports science. 1999; N 17: 697-711. DOI: 10.1080/026404199365560.

15- Shahbazi, Mehdi, Tahmasebi, Shahrzad, Motashari P, Ebrahim, Determining factor validity and reliability of the Persian version of the performance strategy test, Journal of Motor Behavior, 2014; 17: 15-42 .[Persian]

16- Momeni, Fereshteh, Shahidi, Shahriyar, Motaei, Fereshteh and Yadri, Mahmoud, Psychometric properties of the Persian version of the SelfCompassion Scale, Contemporary Psychology. 2013; 8 (2): 40-27.[Persian]

17- Tabachnick, B. G., \& Fidell, L. S. Using multivariate statistics. Allyn \& Bacon/Pearson Education. 2007.

18- Mehri, s., Pooraghai Ardekani, Z. The prediction of counterfactual thinking according to mood and optimistic outlook: study of Iranian men athletic league. Journal of Sport Psychology Studies, 2017; 20: 85-96.[Persian]

19- Ferguson, L. J., Kowalski, K. C Mack, D. E. \&. Sabiston, C. M. Exploring self-compassion and eudemonic well-being in young women athletes. Journal of Sport \& Exercise Psychology, 2014; 36: 203-216. doi: 10.1123/jsep.2013-0096.

20- Birrer. Daniel. Röthlin, P., \& Morgan, G. Mindfulness to enhance athletic performance: Theoretical considerations and possible impact mechanisms. Mindfulness. 2012; 3(3): 235-246.

21- Reis, N. A., Kowalski, K. C., Ferguson, L. J., Sabastian, C. M., Sedgwick, W. A., \& Crocker, P. R. E. Self-compassion and women athletes' responses to emotionally difficult sport situations: An evaluation of a brief introduction. Psychology of Sport and Exercise. 2015; 16: 18-25. doi: 10.1016/ j. psychsport.2014.08.011. 